\title{
Das Future Skills Triple Helix-Modell der Handlungsfähigkeit in emergenten Praxiskontexten
}

Im Design der qualitativen sowie quantitativen Studien, die in die Konstruktion der Future Skills eingeflossen sind, wurden Methoden, Theorien und Datenquellen miteinander trianguliert, um möglichst reich haltige Rekonstruktionen von Future Skills sowie derjenigen Bedingungen zu ermöglichen, welche sie hervorbringen. Dabei ging es vor allem darum, das qualitativ Neue aufzuspüren, was das Entstehen von Future Skills ausmacht.

In der Datenanalyse war es möglich, zusätzlich zur Ermittlung von einzelnen Future Skills auch diejenigen Faktoren zu identifizieren, die den Future Skills zugrunde liegen. Zudem zeigen die Daten die unbedingte Notwendigkeit ständigen Weiterlernens, um den stetigen Anpassungsprozess zu meistern, mit dem Mitarbeiterinnen und Mitarbeiter in hochemergenten Kontexten von Future Organisations handlungsfähig werden und bleiben. Eben solchen Organisationen, die bereits ein weit entwickeltes und explizit formuliertes Verständnis für die Förderung von Handlungskompetenz aufweisen. Die Ergebnisse erlauben Rückschlüsse auf jene individuellen Fähigkeiten und Skills zu ziehen, die in der Zukunft nötig sein werden, um mit den Herausforderungen der Arbeitswelt umgehen zu können.

Zusätzlich erlauben die Daten auch eine modellhafte Rekonstruktion des Bedingungsgefüges, in welchem Future Skills entstehen. Dabei ging es darum, regelhaft Veränderungen und Zusammenhänge zu identifizieren, die systemisch in Organisationen wirken und zu eben den neuen Anforderungen führen, die wir hier als Future Skills bezeichnen. Aufgrund der dreipoligen Struktur bezeichnen wir das so entstehende Modell als das „Future Skills Triple Helix-Modell der Handlungsfähigkeit in emergenten Praxiskontexten“. 


\section{A 2.1 Das Future Skills Triple Helix-Modell}

Zunächst muss angemerkt werden, dass Skill ein Terminus ist, der immer eine Beziehung ausdrückt. Skills erhalten Bedeutung dadurch, dass sie etwas in Beziehung setzen. Kommunikation beispielsweise besteht zunächst nur in der Produktion von Geräuschen, die oftmals Sprache darstellen, die für sich allein genommen jedoch keine Kommunikations-Skills bezeichnen. Erst der Sprechakt, also das in Beziehung setzen zu einer Situation oder anderen Person mittels Sprache, macht eine Form des Ausdrucks notwendig, welche wir dann als angemessen oder befähigt wahrnehmen oder bezeichnen können. Kommunikations-Skills sind also von ihrem reinen Handlungsablauf zunächst nicht bedeutungsvoll. Kommunikationsfähigkeiten einer Person in einer gekonnten (skillfull) Weise in Relation zu etwas oder jemandem erhalten erst Bedeutung durch den Kontext ihrer Handlung. Dabei steht - um im Beispiel zu bleiben - nicht immer nur eine andere Person, etwa eine Dialogpartnerin im Mittelpunkt. Kommunikation kann durchaus auch Beziehungnahme zu sich selbst und der eigenen Position oder zu einem bestimmten Gegenstand - etwa dem Thema - sein.

In den empirischen Daten der Future Skills Studie lassen sich drei solche Relationen rekonstruieren: Eine handelnde Person kann Future Skills in Bezug auf sich selbst entwickeln, kann diese in Bezug auf den Umgang mit einer Aufgabe, einem Thema oder einem Gegenstand, den sie bearbeitet, entwickeln oder in Bezug auf die organisationale Umwelt, also das soziale System. In der Rekonstruktion der Daten und mit Rückgriff auf die erkenntnistheoretischen Grundpositionen der Subjekt-Objekt-Spaltung und der Aufteilung der Objekt-, der Gegenstandswelt in eine gegenständliche und eine soziale, attribuieren wir diesen einen Subjekt-, Objektund (sozialen/ organisationalen) Weltbezug. Es entsteht eine dreipolige Relation, wobei jeder Pol in Relation zum jeweils anderen steht. In Bezug auf Handlungen in hochemergenten Kontexten sind damit in jeder beliebigen Handlung immer alle drei Pole und deren Beziehung untereinander bestimmend. Aufgrund der engen Verwobenheit aller drei Pole und ihrer aufeinander bezogenen Integration bezeichnen wir dieses Konzept im Rückgriff auf das biologische Konzept der DNA als Triple Helix-Modell der Future Skills, bzw. als Future Skills Triple Helix-Modell. Das so entstehende Konzept ist geeignet, zur formalen Beschreibung von Handlungen in hochemergenten Kontexten. Wie gehandelt wird, hängt also ab von der inneren, eigenen Subjektverfasstheit in Bezug zur Handlung, es hängt zugleich ab von dem individuellen Fähigkeitskonzept in Bezug auf einen Gegenstand in Bezug zur Handlung und es hängt ab von dem individuell-sozialen Bezug des Handelnden Subjekts in Bezug zur Handlungssituation. Alle drei Bezüge stehen wiederum in relationalem Bezug miteinander. Das bedeutet, dass jede Handlung in einem Kontext, in dem Future Skills zum Tragen kommen, das Resultat des dreifachen Zusammenspiels ist. 
Diese Strukturierung ermöglicht innerhalb der Future Skill Konstrukte, eine Binnenstruktur zu identifizieren. Sie erlaubt eine Einteilung von Future Skills in Bezug auf die Relation, auf die sie sich bezieht. In Beantwortung der Frage, ob es sich eher um eine subjektive, auf sich selbst bezogene Fähigkeit (beispielsweise selbstgesteuertes Lernen, Selbstkompetenz), eine auf einen Gegenstand oder eine Aufgabe bezogene Fähigkeit oder um eine auf die soziale, organisationale Umwelt bezogene Fähigkeit handelt, lassen sich die Future Skill Konstrukte in drei Bereiche einteilen und binnendifferenzieren. Als Klassifizierungskriterium dient dabei das Ziel der Relation - ob es auf ein Subjekt (Individuum zu sich selbst), Objekt (Individuum zu einem bestimmten Objekt, beispielsweise einer Aufgabe) oder die Umwelt (Individuum zur sozialen Umwelt) - bezogen ist (siehe Abbildung 5):

1. Beziehung eines Individuums zu sich selbst in der Gegenwart, Vergangenheit oder Zukunft (Subjekt- oder Zeitdimension), ${ }^{6}$

2. Beziehung eines Individuums zu einem bestimmten Objekt (Objektdimension) oder

3. Beziehung eines Individuums zu einer Person oder einer Gruppe in der Welt (soziale Dimension).
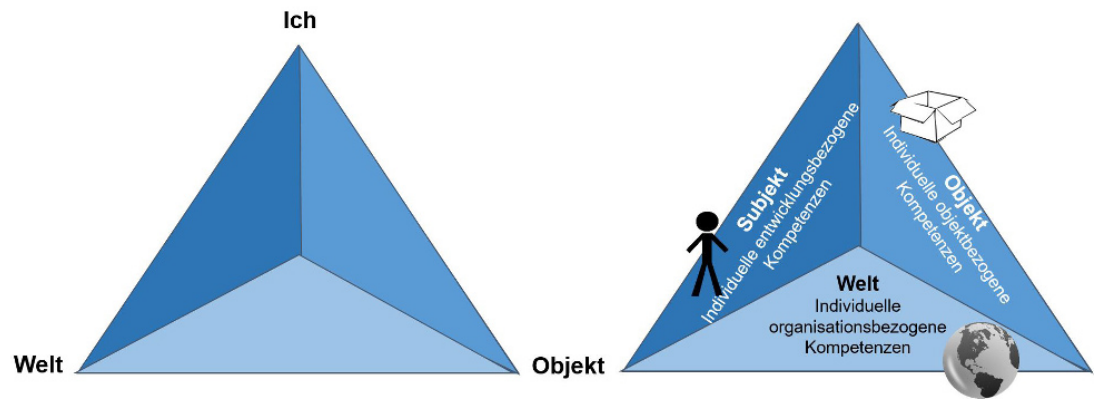

Abb. 5 Dreiteilige Klassifizierung von Future Skills

6 Der Begriff „Zeitdimension“ geht darauf zurück, dass sich Subjekte nur in der Zeit wahrnehmen können, also in Bezug auf etwas Vergangenes, etwas gerade Passierendes oder etwas zukünftig Vorgestelltes. 


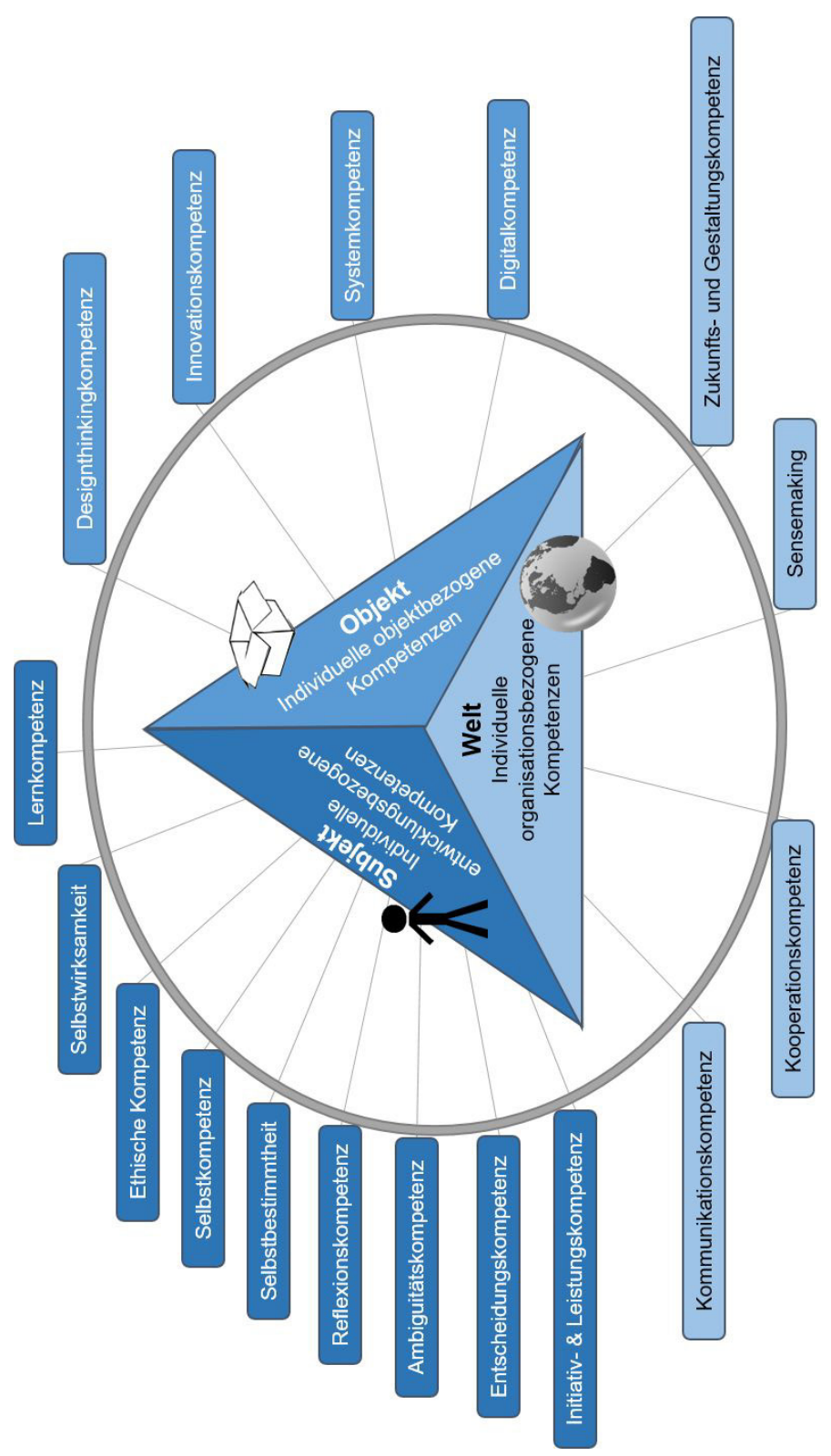

Abb. 6 Future Skills Gesamtschau - Zuordnung zu drei Dimensionen 
Diese dreigliedrige Unterteilung ist in der Philosophie der Bildungswissenschaften tief verankert (z. B. Dewey und Bentley in seinem Aufsatz „Knowing the Known“, Dewey \& Bentley 1949), geht in der Aktualität aber maßgeblich auf Meder (2007, auch Roth (1971)) zurück, der eine fundamentale, konstitutive Struktur für Bildung als einer strukturell-dreigliedrigen Beziehung aufstellt. Für das Future Skills Konzept ergibt sich daraus eine dreidimensionale Aufgliederung: Future Skills beziehen sich also (1) gemäß der Zeit- oder Subjektdimension entweder auf individuell entwicklungsbezogene Aspekte des handelnden Subjekts (beispielsweise die Fähigkeit zur Selbstreflexion in Bezug auf etwas in der Vergangenheit Erlebtes oder ethische Kompetenz), oder beziehen sich (2) auf den Umgang mit einem Gegenstand, einem Objekt, etwa einem Thema, einer Aufgabe (beispielsweise Design Thinking Skills) oder aber (3) auf die soziale Umwelt bzw. die Organisation, in der das Individuum handelt (beispielsweise Kooperations- oder Kommunikationskompetenzen). Subjekt, Objekt oder Welt-/ Organisationsbezug spannen somit die Kompetenzfelder auf, in denen sich Future Skills verorten lassen. Abbildung 6 zeigt die Aufgliederung der Future Skills in die unterschiedlichen Kompetenzfelder.

Alle drei Dimensionen stehen wiederum miteinander in Verbindung und beeinflussen sich wechselseitig. So wirkt beispielsweise die Kompetenz zur Selbstreflexion nicht nur auf die subjektive Entwicklung eines handelnden Individuums, sondern auch auf die Kommunikations- und Kooperationsfähigkeit (soziale oder Organisationsdimension) und wiederum auf die Systemkompetenz eines Individuums (Objektdimension). Insofern sind an jeder Handlung unterschiedliche Future Skills gleichermaßen beteiligt (siehe Abbildung 7). Die drei Dimensionen formen so die Future Skill Triple Helix-DNA in der die drei Skill Dimensionen bei konkreten Handlungen zusammenwirken. Sie ermöglichen ein besseres Verständnis der Faktoren, die zukünftige Handlungsfähigkeit ausmachen. 


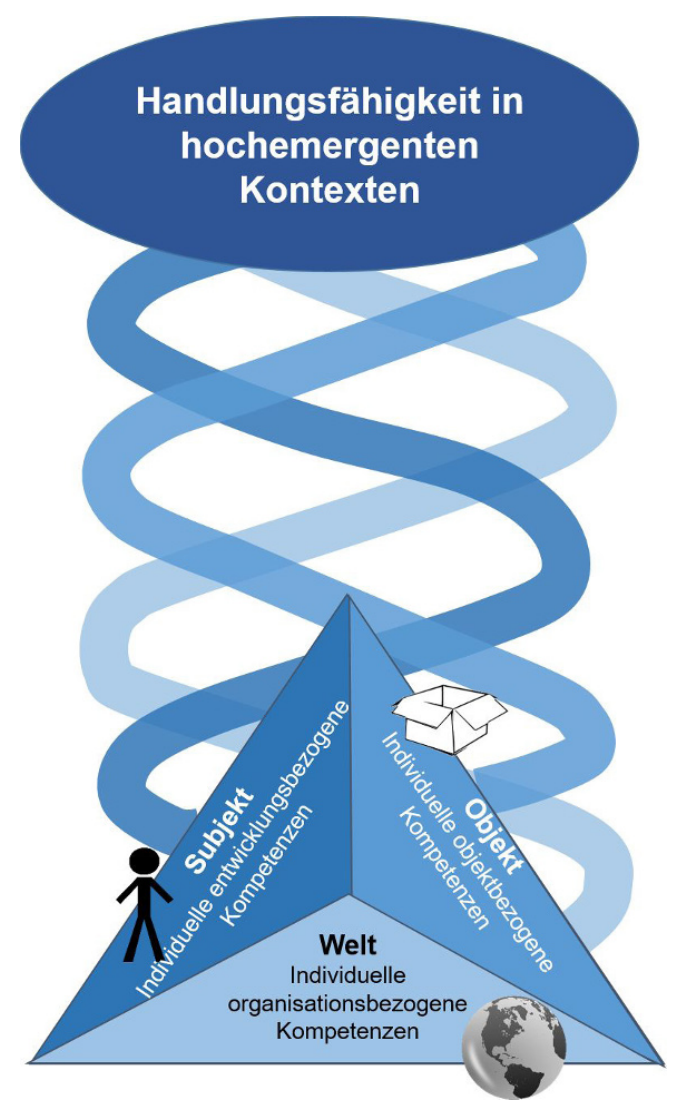

Abb. 7 Triple Helix-Konzept der Future Skills

Das Rückführen der Future Skills auf drei konstitutive Komponenten erlaubt auch eine Aufklärung der Ursachen, die Future Skills so bedeutsam machen. Die empirischen Analysen der Interviewdaten zeigen, dass in jeder der drei Dimensionen Veränderungsprozesse - im Folgenden als Shift bezeichnet - und Verschiebungen ablaufen. Dabei wird deutlich, dass sich ein klarer Wandel im Hinblick auf die Natur derjenigen Fähigkeiten abzeichnet, die für Individuen und deren Handlungsfähigkeit in zukünftigen Arbeits- und Lebenskontexten bedeutsam sind. Künftige Skill-Anforderungen können somit von denen der Vergangenheit und auch zum Teil der gegenwärtigen klar unterschieden werden. 


\section{A 2.2 Shift 1 - Weniger Standardisierung und mehr Selbstorganisation}

Die These von Voß und Pongratz (1998) zum Arbeitskraftunternehmer, von Hitzler und Honer zur Bastelbiographie (1994) und auch von Ulrich Beck (1986) zur Risikogesellschaft legt eine immer geringere Standardisierung von Erwerbsbiographien nahe. Daraus folgt eine stärkere Selbstkontrolle des Individuums in Bezug auf ihre/seine Erwerbsbiographie. Diese Selbstkontrolle der Navigation von einem Job zum nächsten, aber auch von einer Position innerhalb eines Berufes zur nächsten, bzw. von einem Beruf zum nächsten, zeigt sich in den Future Skills Daten auch auf einer Mikroebene. ${ }^{7}$ Auf dieser Ebene lassen sich für die Binnenanforderungen innerhalb von Arbeitsprozessen Fähigkeiten rekonstruieren, die nahelegen, dass es einen Wandel gibt, der weniger vorgegebene Arbeitsstrukturen und mehr Selbstorganisation erfordert. Diese Betonung von Selbstkompetenzen drückt sich in der Rekonstruktion der Daten aus, in denen die Befragten betonen, dass Handlungskontexte in Organisationen sich immer schneller wandeln, sowohl strukturell (in der Organisation) als auch inhaltlich (im Aufgabenbereich) und sozial (im Umfeld). Es wird deutlich, dass dabei durch Individuen immer stärkere individuelle Anpassungsleistungen an neue Handlungskontexte erbracht werden müssen. Diese entstehen oftmals im Rahmen von emergenten Prozessen und sind nur schwer plan- oder vorhersehbar. Die notwendigen Fähigkeiten, die als Future Skills von den Befragten gefordert werden, haben die Aufgabe, diese Anpassungsleistung zu ermöglichen. Dabei wird deutlich, dass ein produktiv-antizipierender Umgang mit sich wandelnden Handlungskontexten eine immer stärkere Bedeutung erfährt, damit nicht kompensatorische Maßnahmen im Vordergrund stehen, die etwa bei

7 Nachtwey (2016) beschreibt den Wandel auf dem Arbeitsmarkt folgendermaßen: Für die Arbeitnehmerinnen und Arbeitnehmer war mit dem Normalarbeitsverhältnis Vorhersehbarkeit des Lebensverlaufes und relative soziale Sicherheit verbunden. Lediglich ca. 10 \% der Beschäftigten arbeiteten zu Beginn der 1970er Jahre in Teilzeit. Dominierte in den 1970er-Jahren noch das sichere Normalarbeitsverhältnis die ökonomische Szenerie, so sind in Deutschland im Jahr 2011 lediglich $28 \%$ der westdeutschen Beschäftigten in privatwirtschaftlichen Firmen mit Branchentarifverträgen beschäftigt (Gundert \& Hohendanner 2011). Im Jahr 1998 waren es noch $39 \%$ (ebenda). Zudem hat sich in einzelnen Branchen das Leiharbeitsverhältnis von der Ausnahme zur Regel gewandelt. In der Lebensmittelindustrie besitzt in Deutschland zurzeit nur jede, bzw. jeder zehnte Beschäftigte einen regulären Arbeitsvertrag (ebenda). Aber auch im Segment der Höherqualifizierten dreht sich die Deregulierungsspirale mit Auswirkungen für die Beschäftigten. Gerade bei IT-Spezialistinnen und -spezialisten nehmen die Werkverträge zu und die Praxis des Crowdworkings verdrängt in der Software- und Autoindustrie fest definierte Tätigkeiten immer häufiger (ebenda). 
Verlust der Handlungsfähigkeit aufgrund von sich ändernden Handlungskontexten darauf abzielen, Handlungsfähigkeit wiederherzustellen. Vielmehr geht es darum, Akteurinnen und Akteure zu befähigen, bereits im Verlauf der Änderungsprozesse neue Handlungskontexte produktiv mitgestalten zu können. Future Skills haben dabei die Aufgabe, Akteurinnen und Akteure zu befähigen, selbstorganisiert handlungsfähig zu sein. Sogenannte Selbstkompetenzen wie beispielsweise Selbstwirksamkeit, Selbstbestimmung, Selbstkompetenz, Reflexionskompetenz und auch das selbstgesteuerte Lernen, ermöglichen es den Individuen, die notwendigen Anpassungsvorgänge in hochemergenten Kontexten produktiv leisten zu können.

\section{A 2.3 Shift 2 - Vom Fachwissen zur Handlungskompetenz}

Ein zweiter Shift, der sich aus den Interviewdaten ergibt, ist der Wandel von der ursprünglich hohen Bedeutung des Fachwissens hin zu einer eher als generisch beschriebenen Handlungskompetenz. Dabei fassen wir in Anlehnung an Erpenbeck (2012) Handlungskompetenz grundsätzlich als die Disposition zur zielgerichteten Handlungsfähigkeit in komplexen und unbekannten Problemsituationen. In Kapitel B 1.2.3 Kompetenz wird auf die besondere Bedeutung von Handlungskompetenz ausführlicher eingegangen. In Anlehnung an Baackes et al. (1991) Kompetenzdimensionen, die er wiederum aus dem Konzept der kommunikativen Kompetenz in Anlehnung an Chomsky (1981) entwickelt und die er für den Bereich der Medienkompetenz ausführt, stehen dabei vier Dimensionen im Mittelpunkt, die den hier beschriebenen Shift gut veranschaulichen können. Die ursprünglich für den Bereich der Medienkompetenz und des Umgangs mit Medien entwickelten Kompetenzdimensionen (in Anlehnung an Baacke, zitiert nach Vollbrecht 2001: 56) werden dabei allgemein auf Handlungsfähigkeit in emergenten Kontexten bezogen, da sich an ihnen der Shift auch im Kompetenzverständnis gut veranschaulichen lässt (siehe Abbildung 8):

- Die Wissensdimension mit einer informativen und einer instrumentell qualifikatorischen Dimension,

- die Dimension der Wissensanwendung mit einer eher rezeptiven und einer eher interaktiven Komponente,

- die Gestaltung von Neuem mit einer innovativen und einer kreativen Komponente und

- die Kritikfähigkeit in Bezug auf einen Wissensbestand mit einer analytischen, einer reflexiven (hier selbstbezogenen) und einer ethischen Komponente. 
Über die Erkenntnis hinaus, dass Future Skills eher Handlungskompetenz verlangen und nicht mehr nur reines Fachwissen ausreicht, erlaubt es das Modell wesentlich präziser zu rekonstruieren, welche Dimensionen von Kompetenz im Future Skills Modell ausgeprägt sind. Dabei wird in den Interviews deutlich darauf hingewiesen, dass Future Skills vor allem die Entwicklung der Gestaltungs- und der kritischen Dimension von Kompetenz notwendig machen, wie in Abbildung 8 veranschaulicht. Individuen konnten sich in der Vergangenheit darauf beschränken, Wissen, Methoden und Tools anzuwenden; künftig wird es aber zunehmend wichtiger werden, neues Wissen, Methoden und Tools originell und auf kreative Art und Weise zu entwickeln.
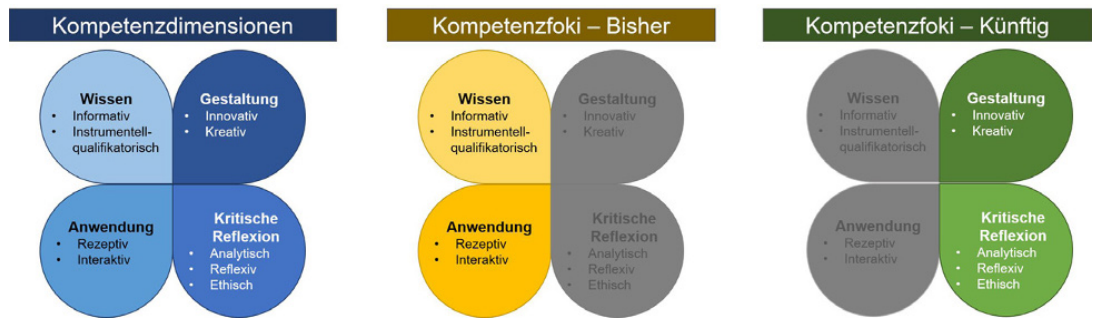

Abb. 8 Änderung bei objektbezogenen Kompetenzen

\section{A 2.4 Shift 3 - Von hierarchischen zu vernetzten Organisationskontexten}

Ein dritter Wandel bezieht sich auf ein sich generell wandelndes Organisationsumfeld von hierarchischen Ablauforganisationen hin zu vernetzten und agilen Organisationen. Der sich hier vollziehende Wandel wird in den Interviewdaten deutlich beschrieben: Während Organisationen in der Vergangenheit in klaren Strukturen und Managementprozessen organisiert waren, werden die Organisationen der Zukunft in fluideren Strukturen organisiert werden, die schnelleren und grundsätzlicheren Änderungen unterliegen. Abbildung 9 illustriert, dass dabei konkurrierende Pole einander gegenüberstehen, bei denen die bisherigen Strukturen und Abläufe von klar definierten Managementstrukturen zukünftig eher durch agile Abläufe und ein Ermöglichungsmanagement ersetzt werden. Die klassische bisherige Ablauforganisation wird hingegen durch vernetzte Strukturen geprägt sein, in denen klar definierte Prozesse sich häufiger weiterentwickeln und Organigramme und 
Zuständigkeiten sich schneller wandeln. Beziehungsmanagement wird dabei ein zunehmend wichtiger Faktor. Der gesamte Bereich informeller Eigeninitiative ist ein wichtiger Bestandteil von organisationalem Erfolg und ein wesentlicher Future Skill ohne den die Steuerung von Organisationen zukünftig ineffizient wird. Die Befragten drücken aus, dass in Future Organisations zentrale Steuerungsansätze in Organisationen immer weniger zielführend sind und stattdessen beteiligungsorientierte Zielfindungsprozesse immer mehr an Bedeutung zunehmen.

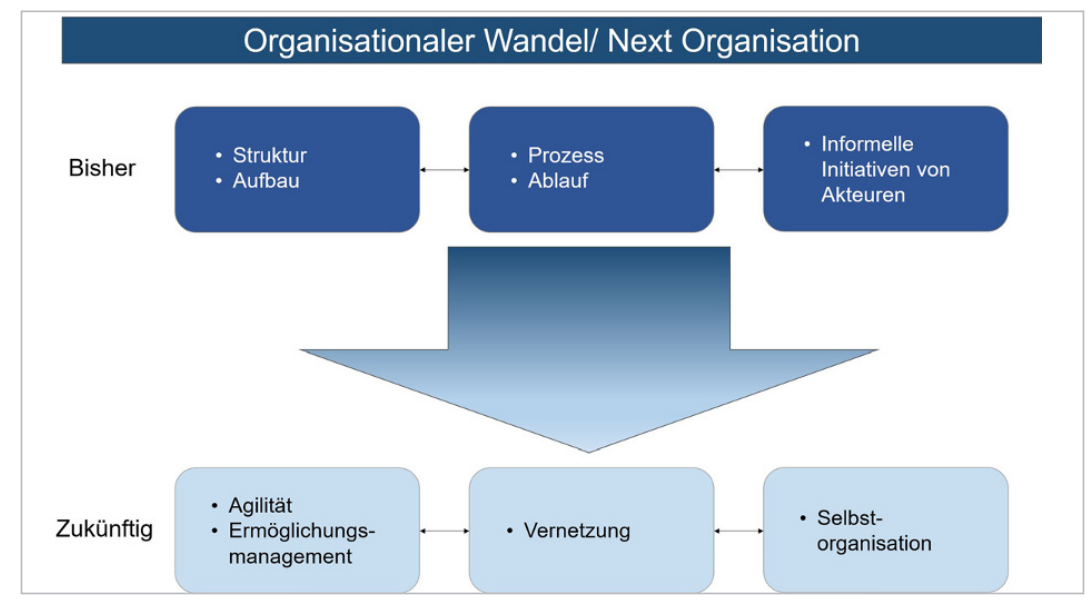

Abb. 9 Organisationaler Wandel in Future Organisations

\section{A 2.5 Zusammenfassung und Fazit}

Abbildung 10 verortet die beschriebenen drei Shifts in einer zusammenfassenden Grafik an den drei Dimensionen des Triple Helix-Modells. Alle drei Dimensionen stehen miteinander in Interaktion und sind keine bloßen Ausdrücke isolierter Skill-Bereiche. Subjektive Aspekte beeinflussen sowohl die Perspektive auf objektive Aspekte, als auch auf soziale Aspekte, die wiederum Auswirkungen auf subjektive und objektive Aspekte haben. Das vorgestellte Future Skills Modell geht damit über ein statisches, Future Skills lediglich definierendes und aufzählendes Modell hinaus. Außerdem basiert das Modell zwar auf der Annahme, dass digitale oder technische Skills künftig zweifelsohne eine wichtige Future Skills Zutat sein werden, sieht diese Skills aber nicht als alleinig ausreichend an. Der wirkliche Wert dieser Skills liegt daher 
vor allem in der persönlichen Entwicklung von Dispositionen, die das Individuum zu selbst-organisiertem Handeln in einer definierten Domäne befähigen können.

Das hier präsentierte Future Skill Konzept basiert auf den folgenden drei unterscheidbaren Momenten theoretischer Reflexion:

1. Skills werden als relationale Konzepte verstanden, die mittels der dreidimensionalen Struktur analog zum strukturellen Bildungsbegriff beschrieben werden können.

2. Skills werden als Kompetenzen im Sinne Erpenbecks (2010) verstanden und es wird betont, dass Kompetenzen Dispositionen darstellen, um in komplexen, unbekannten Zukunftskontexten agieren zu können.

3. Future Skills werden in Bezug zu Verschiebungen innerhalb der unterschiedlichen Bestandteile des theoretischen Rahmens verstanden und können mithilfe der 17 identifizierten Future Skills Profile beschrieben werden.

Mit dieser theoretischen Rahmung wird das vorliegende Future Skill Konzept in den Bildungswissenschaften verankert. Anstelle einzelne Future Skills in Listenform additiv zusammenzustellen, gibt der hier gewählte Ansatz eine einheitliche und präzise Richtung für die Skill-Terminologie vor und erlaubt es, genau zu fassen, was mit Future Skills gemeint ist.

Zusammenfassend kann festgehalten werden, dass das Future Skill Modell die Erklärungskraft hat, eine Reihe von Future Skills anhand eines klar strukturierten und beschreibbaren Dimensionssets abzubilden:

1. Die erste Future Skill Dimension stellt die subjektive (oder auch Zeit-) Dimension der Future Skills Profile dar. Diese bezieht sich auf die subjektiven, personalen Fähigkeiten eines Individuums, sich so anzupassen und zu entwickeln, dass es produktiv an der Arbeitswelt von morgen teilhaben und diese aktiv mitgestalten sowie sich selbst in Gemeinschaften organisieren kann, um mit künftigen Herausforderungen effektiv umgehen zu können. Diese Dimension beinhaltet neun Future Skills Profile.

2. Die zweite Future Skill Dimension bezieht sich auf die Fähigkeit von Individuen in selbst-organisierter Art und Weise in Bezug auf ein Objekt, eine Aufgabe oder eine bestimmte Thematik zu handeln. Diese Dimension begreift Wissen weiterhin als zentral, plädiert aber dafür, es gedanklich auf die nächste Stufe zu heben und es zusammen mit Motivation, Werten und Absichten zu denken. Damit wird Wissen mit einer neuen Facette aufgeladen, die die Wichtigkeit selbst-organisiert in unterschiedlichen Wissensfeldern zu agieren, betont. Es ist nicht mehr allein die Menge des Wissens, die zählt, sondern vielmehr die Frage, wie mit diesem 
Wissen produktiv umgegangen werden kann, um anstelle von Expertise zu Professionalität zu gelangen. Diese Dimension vereint vier Future Skills Profile.

3. Die dritte Future Skill Dimension bezieht sich auf die Fähigkeiten eines Individuums im Hinblick auf seine/ ihre soziale und organisationale Umwelt und die Gesellschaft selbstorganisiert zu agieren. Hierbei wird betont, dass auf Individuen eine Doppelrolle zukommt: Einerseits sind sie Kuratorinnen und Kuratoren ihrer sozialen Mitglieds-Portfolios in unterschiedlichen organisationalen Sphären, während sie andererseits und gleichzeitig organisationale Räume selbst kreieren und organisationale Strukturen neugestalten, um sie zukunftsbeständig zu machen. Vier Future Skill Profile sind unter dieser Dimension zusammengefasst.

Die geänderten Grundbedingungen von Arbeit und Lernen führen zu einer Verschiebung der Zutaten, die für die Fähigkeit erfolgreichen Handelns notwendig sind. Im Konzept der Future Skills werden drei Bestandteile als wesentliche Bezugspunkte angesehen, die auf ein relationales, strukturelles Verständnis von Bildung zurückgehen. Darin wirkt der Prozess des Erwerbs von Future Skills in dreifacher Weise:

1. Bildung und Lernen als Prozess der individuellen Weiterentwicklung und Selbstbildung (Ausbildung eines Verhältnisses zu sich selbst)

2. Bildung als Prozess der Aneignung eines bestimmten Gegenstandes, Fachgebietes oder Wissensbestandes (Ausbildung eines Verhältnisses zu einem Objekt)

3. Bildung als Prozess der Entwicklung der eigenen Position in einer Gemeinschaft (Ausbildung eines Verhältnisses zur Welt).

Grundsätzlich stehen dabei alle drei Elemente dieses Bildungsbegriffes in einem Zusammenhang. So beeinflusst das eigene Selbstbild, der entwickelte Selbstwert auch die eigene Bezugnahme zu einem Gegenstand oder zur Welt und vice versa.

In zukünftigen Arbeitskontexten, dort also, wo Future Skills immer relevanter werden, kann nun eine Verschiebung beobachtet werden: Unter Bedingungen gröBerer Selbstorganisation wandeln sich die Bedeutung und Zielrichtungen der drei Pole des Future Skills Dreiecks (siehe Abbildung 10). Der Prozess der subjektiven Entwicklung im Sinne der Ausbildung eines Verhältnisses zu sich selbst nimmt einen neuen Stellenwert ein. Er erfordert, dass solche subjektiven Fähigkeiten neu in den Fokus rücken, die etwa als Selbstorganisation, Selbstwert, Selbstkompetenz usw. beschrieben werden können. Dieser Pol weist einen zentralen Stellenwert als Steuerungsinstanz für unser Verständnis von Future Skills auf. Der eher objektbezogene Pol hingegen wandelt sich in seiner Bedeutung dahingehend, dass es zwar auch in zukünftigen Lern- und Arbeitsumgebungen notwendig sein wird, Wissen über Lerngegenstände zu erlangen, dass die subjektive Metadimension 


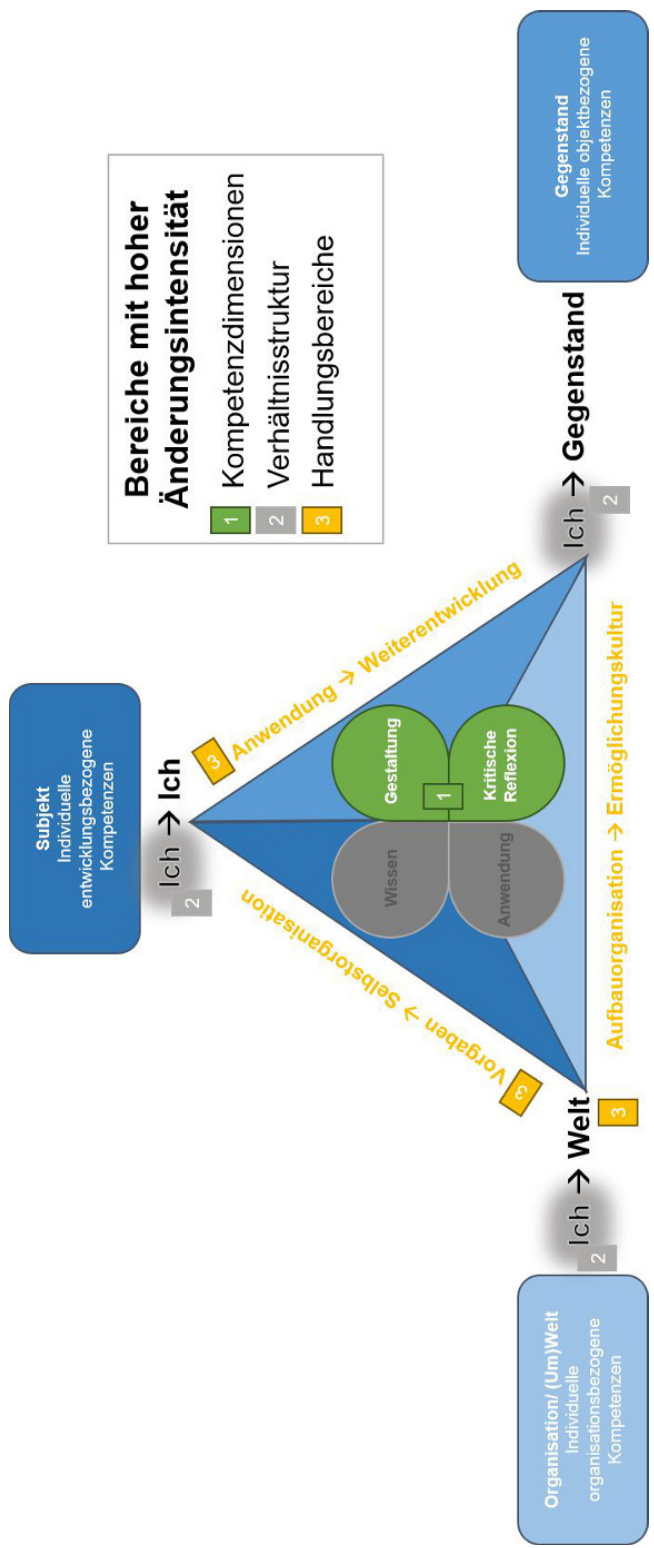

Abb. 10 Kopplung von strukturellem Bildungsmodell und Kompetenzmodell als Erklärungskonzept für Future Skills 
der Aneignung dieser aber über dem tatsächlichen Substrat des Angeeignetseins steht. Es geht also weniger darum Wissensbestände zu akkumulieren, sondern vielmehr darum in der Lage zu sein, Wissen zu finden, einzuschätzen, kritisch zu beurteilen und immer wieder neu den Bezug zur eigenen Position zu reflektieren. Trainings, Weiterbildung und Lehrveranstaltungen müssen sich aus dieser Sichtweise radikal ändern, indem sie zu Reflexionslaboratorien im Sinne Donald Schöns (1983) werden, in welchen nicht das Auswendiglernen und Anhäufen von Wissen im Vordergrund steht, sondern das Ausbilden eigener Handlungsstrategien für komplexe Situationen und die Fähigkeit, subjektive Handlungsstrategien zu reflektieren, zu bewerten und neu zu fassen.

Die Ausbildung eines Verhältnisses zur Organisation, als dritter Pol, steht unter der Zielsetzung, dass das handelnde Subjekt in Bezug auf die Gemeinschaft, die Gruppe, die soziale Struktur, die Organisation und Abteilung bezugsfähig wird. In der Untersuchung zeigt sich, dass dies ein zweiseitiger Prozess ist. Er fordert einerseits das handelnde und lernende Subjekt in neuer Weise, da sich Organisationsstrukturen schnell ändern; andererseits steht die Organisation unter Änderungsdruck in Bezug auf neue Organisations- und Führungskonzepte, da das Verständnis von Organisation nicht mehr länger darin besteht, dass Organisationen überdauernde, festgefügte Strukturen haben, die in Linienhierarchie aufgestellt sind, sondern dass es sich bei Organisationen nun viel mehr um dynamische und komplexe Gebilde handelt, die fluid darauf basieren, dass flexible, agile Individuen in ihnen agieren, die die jeweiligen Strukturen begründen und stetig weiterentwickeln. 